\title{
Paradental Cyst: Report of an Unusual Occurrence with Emphasis on Its Pathogenesis
}

\author{
RohitSinghai ${ }^{1}$, Jyoti D Bhavthankar ${ }^{2}$, Mandakini Mandale ${ }^{3}$, Jayanti Humbe ${ }^{4}$ \\ ${ }^{1}$ (Department Of Oral Pathology and microbiology, Government Dental College And \\ Hospital,Aurangabad/Maharashtra University Of Health Sciences, Nashik, India) \\ ${ }_{2}^{2}$ (Department Of Oral Pathology and microbiology, Government Dental College And \\ Hospital,Aurangabad/Maharashtra University Of Health Sciences, Nashik, India) \\ ${ }_{3}^{3}$ (Department Of Oral Pathology and microbiology, Government Dental College And \\ Hospital,Aurangabad/Maharashtra University Of Health Sciences, Nashik, India) \\ ${ }^{4}$ (Department Of Oral Pathology and microbiology, Government Dental College And \\ Hospital,Aurangabad/Maharashtra University Of Health Sciences, Nashik, India)
}

\begin{abstract}
Paradentalcyst $(P C)$ is an "inflammatory cyst arising on the lateral aspect of a vital tooth as a result of an inflammatory process in the periodontal pocket". Its prevalence varies from 1 to $5 \%$ in all odontogeniccysts, which accounts for its inclusion in the group of rare lesions. The most common site being distal \&buccal aspect of partially or fully erupted mandibular 3rd molars, the present paper mentions a case of unusual occurrence of PC on the mesial aspect of an unerupted mandibular third molar with emphasis on its pathogenesis.

A 16 year old male patient presented with swelling on right side of the face along with pericoronitis associated with 48.Radiographically well-circumscribed unilocular radiolucency was seen in association with mesial root of unerupted 48, provisionally being diagnosed as a cystic lesion. On enucleation histopathology was reminiscent of inflammatory cyst. Correlating the clinical picture the lesion was diagnosed as paradental cyst on mesial-aspect of 48 .
\end{abstract}

Keywords: Inflammatory,Mesial aspect, Odontogenic cyst, Paradental cyst , Third molar.

\section{Introduction}

The paradental cyst is a lesion recognized by WHO (Histological Typing of Odontogenic Tumors 1992) and defined as "an inflammatory cyst arising on the lateral aspect of a vital tooth as a result of an inflammatory process in the periodontal pocket".Literature states paradental cysts as rare, accounting 3-5\% of all the odontogeniccysts ${ }^{[1]}$. Hofrath (1930) was first to report on several cases of jaw cysts located distally to third mandibular molar with pericoronitis as "Marginal wisdom tooth cyst" which Philipsen et albelieved met all the criteria for a paradentalcysts $(\mathrm{PC})^{[2]}$, a term first introduced by Craig $^{[3]}$ in 1976.The cyst occurs in relation with partially/fully erupted mandibular third molars at distal,buccal and rarely mesial aspect.It may also occur although less frequently with the second and first molars. There are rare reports associating PC with premolars ${ }^{[4]}$ or incisors/canines ${ }^{[5]}$. The major clinical feature of the $\mathrm{PC}$ is the presence of a recurring inflammatory periodontal process, usually pericoronitis.There is no concurrence with regards to its pathogenesis. The reduced enamel epithelium and epithelial rests of malassez are believed to have the potential for proliferation in response to inflammatory stimuli and thus give rise to cyst formation.Other hypotheses include an origin from the crevicular epithelium and epithelial remnants of the dental lamina ${ }^{[1,2]}$.

\section{Case Report}

A 16 year old male patient referred to the Department of Oral Pathology with a clinical presentation of slight swelling and stiffness on the right side of the face since 1 month. History was noncontributory.

On extraoral examination slight diffuse swelling of size $1 \times 2 \mathrm{~cm}$ was seen extending from angle of mandible uptotemporomandibular joint area(Fig1).Swelling was afebrile and firm on palpation.Intraoral examination revealed gingival inflammation distal to 47 surrounding the crown of unerupted 48 suggestive of pericoronitis(Fig2).Rest of the mucosa appeared to be normal.On palpation concavity was felt in the lingual cortical bone in the region of 47 and 48 .

Panoramic radiograph showed well circumscribed unilocular radiolucency with sclerotic border mesial to unerupted 48(Fig3).Periodontal ligament space and laminadurawas intact. On CT scan transverse section showedwell circumscribed radiolucent lesion with thinning of lingual cortical plate suggestive of a cystic lesion(Fig4).3D reconstruction image showed perforation of lingual cortical plate in the region between 47 and 48 (Fig5). Aspiration revealed bloody purulent cystic fluid(Fig6). 
Pulp vitality tests with respect to $46 \& 47$ yielded a positive response thus the suspicion of pulpal infection was ruled out.Provisionally diagnosis of a cystic lesion was made.Patientwas advised aexcisional biopsy along with extraction of 48. Gross specimen showed cystic lining attached at cementoenamel junction on mesial aspect of the tooth(Fig7). Histopathological examination revealed a cystic cavity lined by hyperplastic non keratinized stratified squamous epithelium showing leukocyticinfiltration and spongiotic changes in some areas(Fig8).The capsule consisted of fibrous connective tissue presenting hyperemic vessels, areas of hemorrhage ,and inflammatory cell infiltrate(Fig9).Correlating the clinical picture the lesion was diagnosed as paradental cyst on mesial-aspect of 48.Patient underwent a post-operative assessment after 6 months and showed almost complete resolution of the lesion.

\section{Discussion}

The prevalence of PC varies from $1 \%$ to $5 \%$ in all odontogeniccysts ${ }^{[1,3]}$ and we believe its true incidence to be evenhigher.PCs commonly appear on buccalaspects and rarely on the distal aspect ${ }^{[1,2]}$ of partially or fully erupted vital teeth but in this case it was found mesial to $3^{\text {rd }}$ molar. This lesion was first described by Main who used the term "inflammatory collateral cyst". Craig, however, preferred the term "paradental cyst"which was also supported in by Shear, who suggested the term "inflammatory collateral cyst" as being appropriate for those rare cysts that arise in periodontal pockets. While the use of the term inflammatorylateral periodontal cyst has been discouraged since it may be confused with the developmental lateral periodontal cyst. ${ }^{[1]}$ Vedofte et al ${ }^{[6]}$ suggested the term inflammatory paradental cyst to be preferable since inflammation seems to be more important for the developement of the lesion while Stone \& Worth suggested the term " Mandibular infected buccal cyst" for the cyst involving $1^{\text {st }}$ and $2^{\text {nd }}$ mandibular molars.

An explanation as to why the buccalaspect is so often the site of PC can be offered by the fact that the mesio-buccal cusp is the first to break through the oral mucosa and be exposed to the oral environment. ${ }^{[7]}$ Other local anatomical factors like crown form, fissure pattern, adjacent teeth, and gingival architecture may also influence the definite location of the cyst.There is marked prevalence of PC in males. No reasonable explanation can be given for this unusual gender distribution, since there is no known difference in the incidence of impaction or pericoronitisbetween males and females. ${ }^{[1]}$ White persons are affected more frequently than blacks since it is well established that the frequency of third molar impaction and partial eruption is higher in white persons.

There are some factors (like superimposition of anatomical structures, presence of infection, and lesion size and location) that can vary the radiographic presentation of the PC. However, the lesion frequently produces a welldefined radiolucency, mimicking the periapical pathology of the involved tooth. As the inflammatory component is not of endodontic origin, the periodontal ligament space and the lamina dura are usually intact and continuous around the root as was found in this case. ${ }^{[7]}$

\section{Pathogenesis}

While there is little doubt that the paradental cyst arises as a result of a chronic inflammatory stimulus causing proliferation of crevicular and/or odontogenic epithelium, much the same as other inflammatory odontogenic cysts, the exact histogenesis remains uncertain. ${ }^{[1]}$

Craigsuggested that remnants of reduced enamel epithelium(Early epithelial attachment) on the surface of the enamel spur in the furcation of the roots might be induced to proliferate and undergo cystic degeneration. ${ }^{[3]}$ Ackerman ${ }^{[1]}$ also favored its origin from reduced enamel epithelium but suggested that cyst formation occurs as a result of unilateral expansion of the dental follicle secondary to inflammatory destruction ofperiodontium and alveolar bone unlike dentigerous cyst and argued that if the cell rest of malassezwere responsible for this development, then the PCs should have been equally distributed around the root surface.

This suggestion is supported by the frequent radiographic observation of a dilated follicular space distal to the crowns of partially erupted mandibular third molars, the location of the cysts, the attachment of the cysts to the teeth at the cementoenamel junction, and the continuity between cyst lining and reduced enamel epithelium. ${ }^{[1]}$ Since similar evidences were found in our case, we support the hypothesis put forth by Ackerman regarding the pathogenesis of PC.

While accepting that the paradental cyst was an entity, Fowler and Brannon ${ }^{[9]}$ suggested that it may be a variant of the dentigerous cyst or derived from an occluded periodontal pocket. Vedtofte and Praetorius ${ }^{[6]}$ were satisfiedthat the cyst was of inflammatory origin, initiated bya pericoronitis at the time of tooth eruption, and consideredrests of Malassez and reduced enamel epithelium the most likely source of the cyst epithelium.

Colgan ${ }^{[8]}$ studied relation between the angle of tooth impaction \& the location of the cyst and proposed a role for food impaction in the development of the cyst. Quite how the cysts forms after food impaction is not clear. But it can be theorize that the resultant inflammatory process in the soft tissues leads to fluid accumulation within the obstructed 'pocket' by osmotic processes leading to cyst formation. ${ }^{[8]}$ Indirect support for this 
hypothesis comes from the presence of foreign body-type giant cells in some PC cases, which implies a response to material derived from the oral cavity.

\section{Differential Diagnosis}

A dentigerouscyst,developmental lateral periodontal cyst and lateral radicular cyst can be considered as differential diagnoses, depending on radiographic and clinical findings. The relation between dentigerous cysts and paradental cysts is worthy of discussion becausein many centers these lesions may be confused in radiographs \& misdiagnosed.In the dentigerous cyst, expansion of the follicle is the primary event with consequent bone destruction unlike paradental cyst. Also presence of Colgan's sign (the preservation of the distal follicular space in a radiograph) is a useful diagnostic feature to distinguish paradental cysts from dentigerouscysts. There is no simple method of radiographically distinguishing dilated follicular spaces from a paradental cyst. ${ }^{[1]}$ Thus it is suggested that the same criteria be applied as for the dentigerous cyst, that is, a radiolucency in excess of $4 \mathrm{~mm}$ is regarded as a cyst while smaller lesions are considered dilated follicles.

Histologically they are alsodistinct, in contrast to the PC, an epithelium consisting of two to four layers of cuboidal epithelial cells with a flat epithelial-connective tissue interface is generally present in dentigerouscysts. However, the presence of inflammation, hyperplasia and leukocyte exocytosis, similar to that observed in PCs, can be observed. Also the epithelium is most frequently found adhering to the cementoenamel junction. In addition, the bony cavitation of a lateral dentigerous cyst is commonly found around the entire crown of an unerupted tooth. ${ }^{[7]}$ Thus, the diagnosis of a dentigerous cyst in the present case was ruled out through a combination ofhistopathologic and radiographic features.

Another explanation could be that the cysts were secondary infected developmental lateral periodontal cysts. However, this suggestion also seems unlikely, since the average age of patients with developmental lateral periodontal cysts is 50 to 54 years in contrast to the average age of 16 years in the present case. Moreover, the developmental lateral periodontal cyst is mainly localized to the canine and bicuspid region ${ }^{[6]}$ and does not occur as the result of inflammation, but is thought to represent a developmental cyst arising from rests of the dental lamina (rests of Serres).Also vitality of tooth allows exclusion of lateral radicular cyst.

\section{Conclusion}

When paradental cysts are found in the buccal or distal aspects of the roots, their correct diagnosis stays obvious; however at instances that are likely to be rare like their occurrence on the mesial aspects, warrants thorough investigations as they are most likely to be misdiagnosed as dentigerous cysts. As per the literature since years along, two main hypotheses have been suggested regarding their pathogenesis; one as their origin from the reduced enamel epithelium and the other from the rests of Malassez; out of which most of the authors agree at its origin from the reduced enamel epithelium. Moreover the occurrence of paradental cyst in the mesial aspect of mandibular third molar in our case, adds to this agreement. However, there could have been no concrete justification for the same due to the scarcity of reported cases. Thus reporting the occurrences of PCs in both the rare and the not so rare sites is strongly encouraged to precisely come up with the pathogenesis of the same.

\section{References}

[1]. Ackermann et al. The paradental cyst: A clinicopathologic study of 50 cases. Oral Surg1987;64(3):308-312.

[2]. Philipsen et al. The inflammatory paradentalcyst : a critical review of 342 cases from a literature survey, including 17 new cases from authors files. JOralPathol Med 2004;33:147-155.

[3]. Craig etal.Theparadentalcvst. A specific inflammatorvodontogenic cyst. British Dental journal1976 ;141:9-14.

[4]. Morimoto Y, Tanaka T, Nishida I, et al. Inflammatory paradental cyst (IPC) in the mandibular premolar region in children. Oral Surg Oral Med Oral Pathol Oral RadiolEndod. 2004;97:286-93.

[5]. Main DM. Epithelial jaw cysts: a clinicopathological reappraisal. Br J Oral Surg.1970;8:114-25.

[6]. Vedtofte et al The inflammatory paradental cyst. Oral surg oral med oral pathol 1989;68(2):182-188

[7]. Bruno Ramos Chrcanovic ,Brenda Mayra Maciel, Vasconcelos Reis \&BeliniFreire-Maia. Paradental (Mandibular Inflammatory Buccal) Cyst..Head and Neck Patho2011;(5):159-164

[8]. Colgan et al. Paradental cysts: a role for food impaction in the pathogenesis? A review of cases from Northern Ireland.,British Journal of Oral and Maxillofacial Surgery 2002;40: 163-168.

[9]. Craig fowler and Robert Brannon. The ParadentalCyst:AClinicopathologic Study of Six New Cases andReview of the Literature. J Oral MaxillofacSurg1989;(47):243-248.

[10]. Harnisch et al : Clinical Aspects and Treatment of Cysts of the Jaws. Chicago Quintessence, 1974:63-64. 


\section{Pictures}

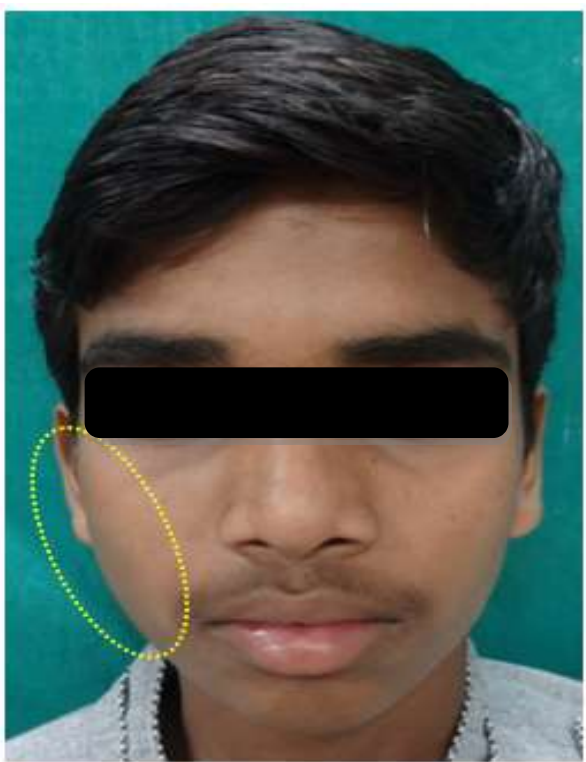

Fig 1: Slight diffuse swellingof size $1 \times 2 \mathrm{~cm}$ with illdefined borders was seen extending from lower border of mandible uptotemporomandibular joint area.

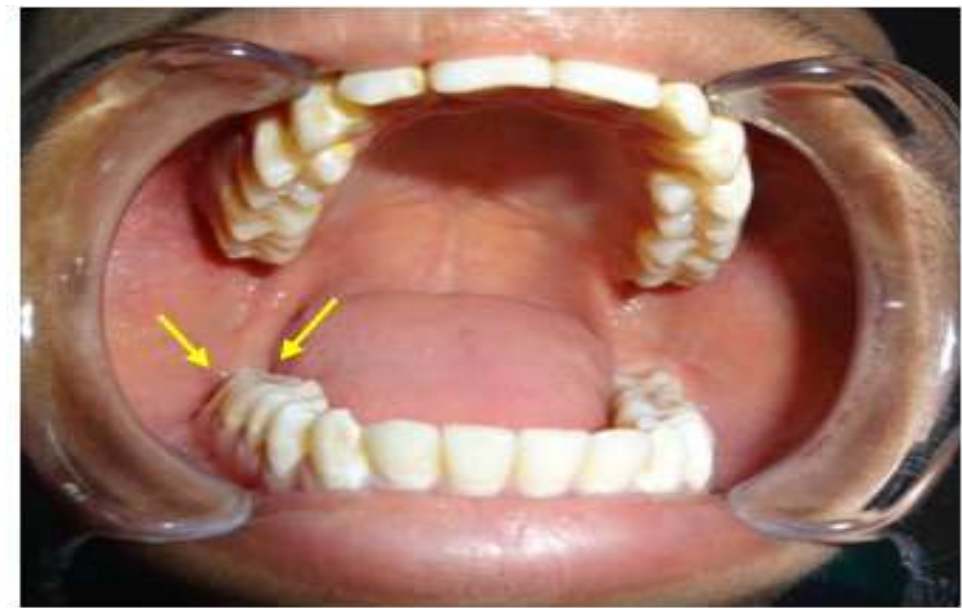

Fig 2 :Gingival inflammation distal to 47 suggestive of pericoronitis.Rest of the mucosa appeared to be normal with no other abnormalities

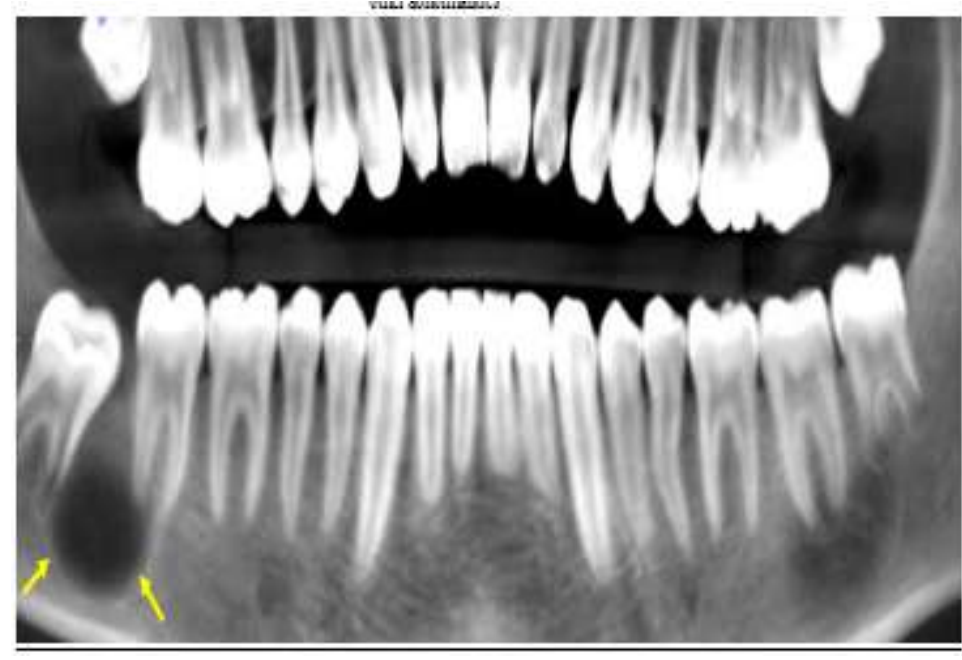

Fig 3: Well circumscribed unilocular radiolucency with sclerotic border mesial to unerupted 48 .Periodontal ligament space and laminadura was intact. 


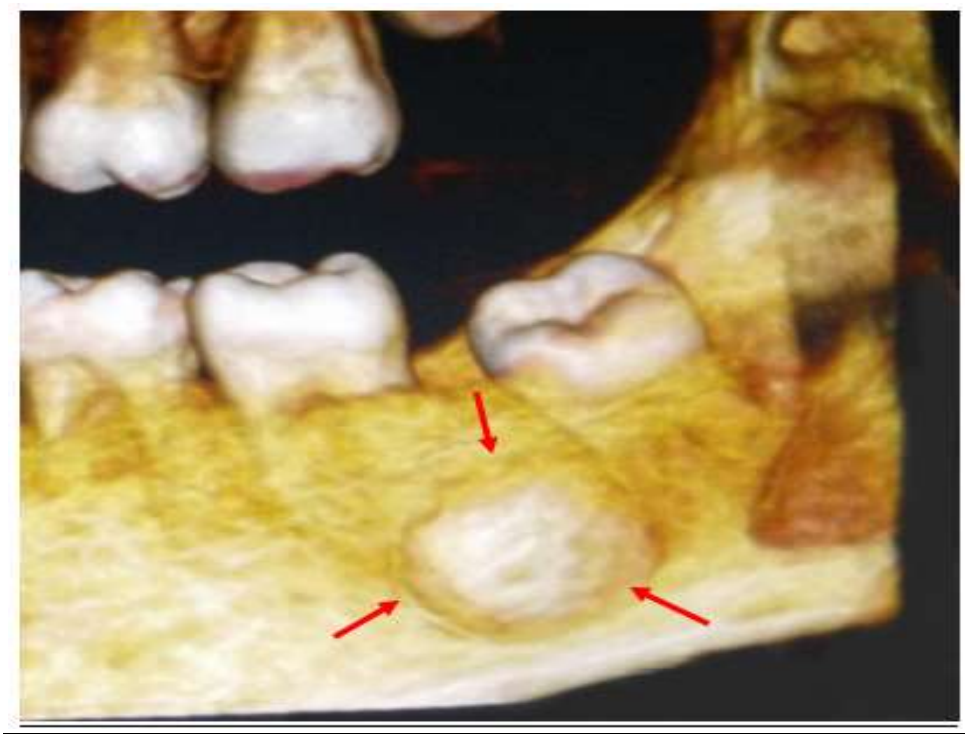

Fig 5: 3D reconstruction image showed perforation of lingual cortical plate in the region between 47 and 48

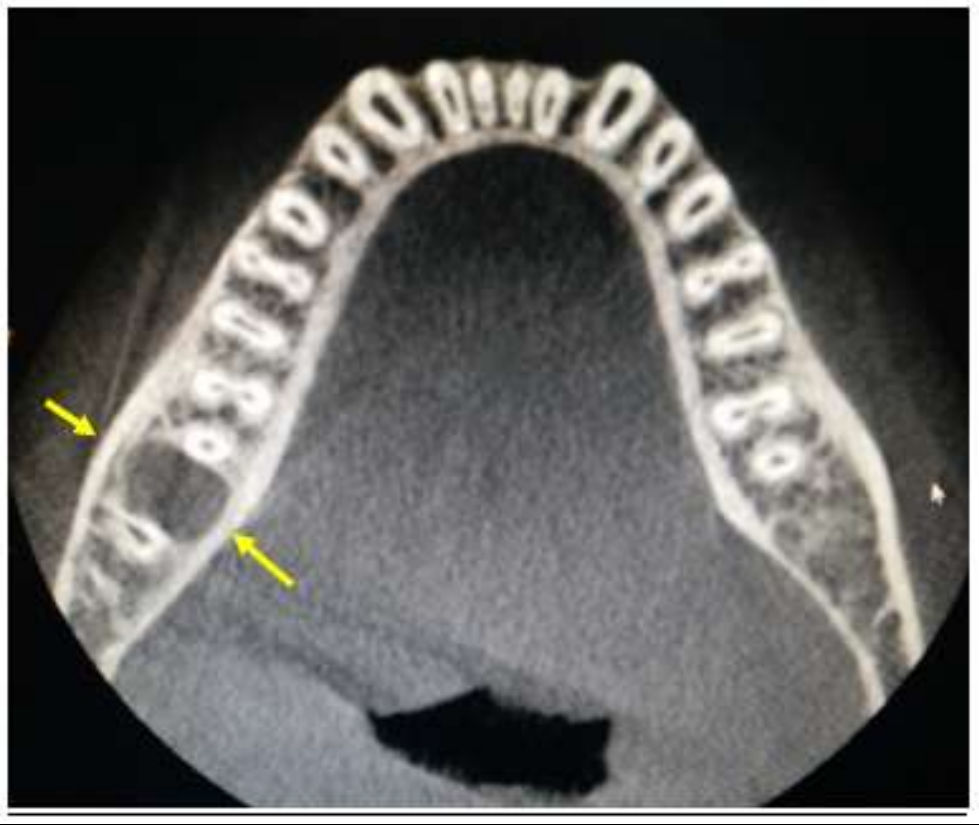

Fig 4:CT scan transverse section shows well circumscribed radiolucent lesion with thinning of lingual cortical plate suggestive of a cystic lesion.

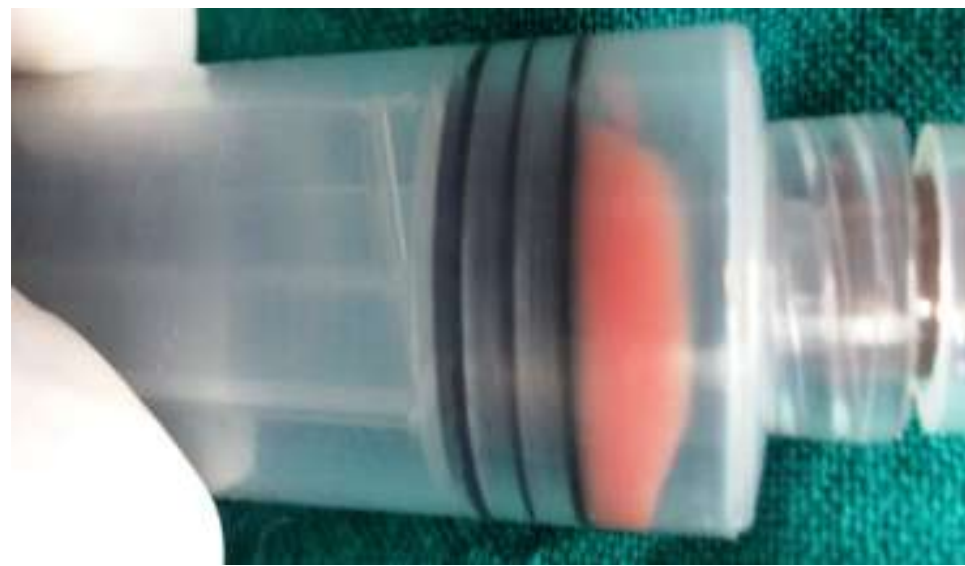

Fig 6 : Aspiration revealed bloody purulent fluid. 


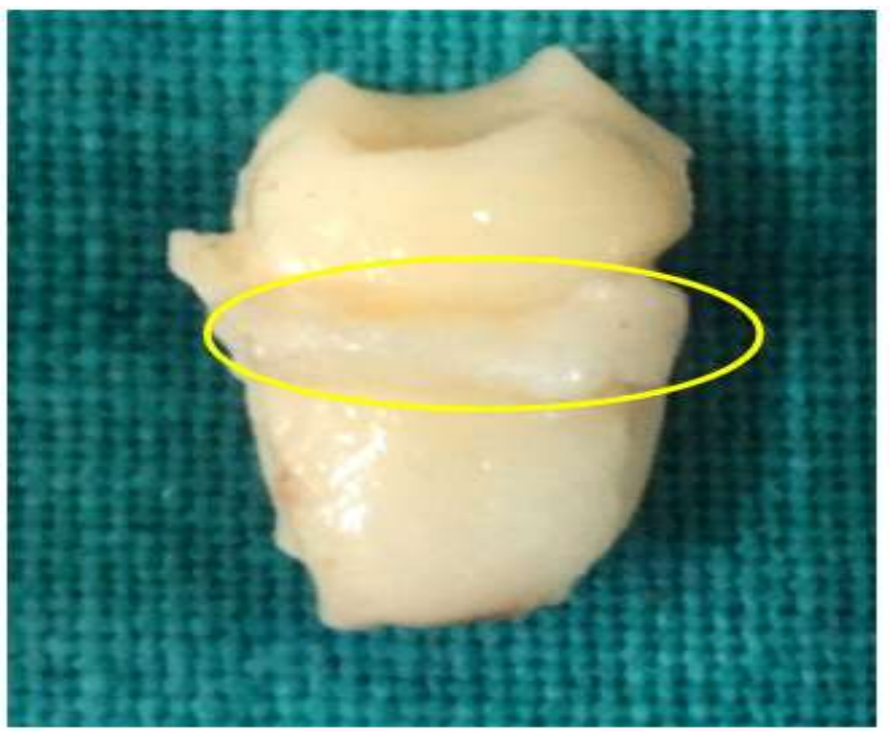

Fig 7 :Gross specimen shows cystic lining attached at cementoenamel junction on mesial aspect of the tooth

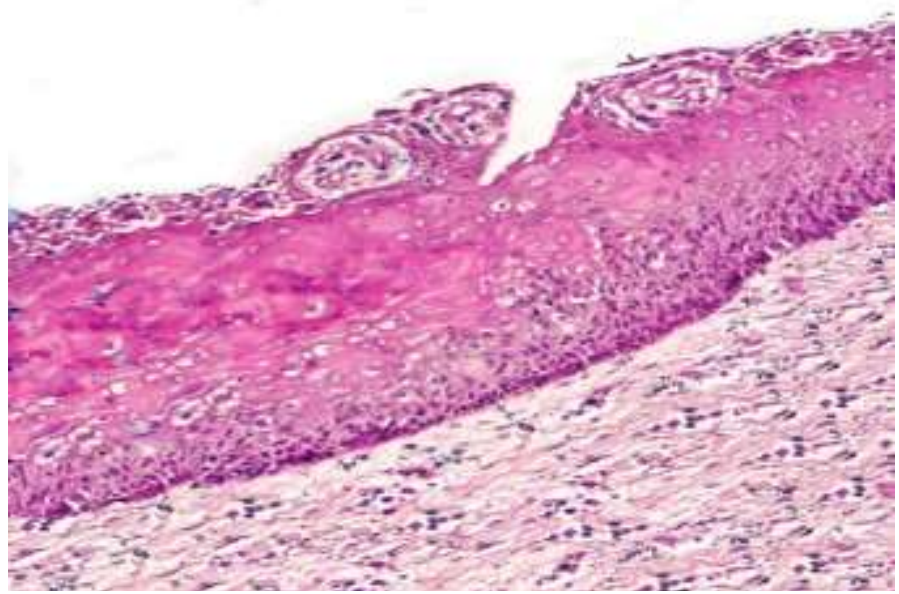

Fig 8: Histopathological examination revealed a cystic cavity lined by hyperplastic non keratinized stratified squamous epithelium showing leukocyte infiltration and spongiotic changes in some areas.

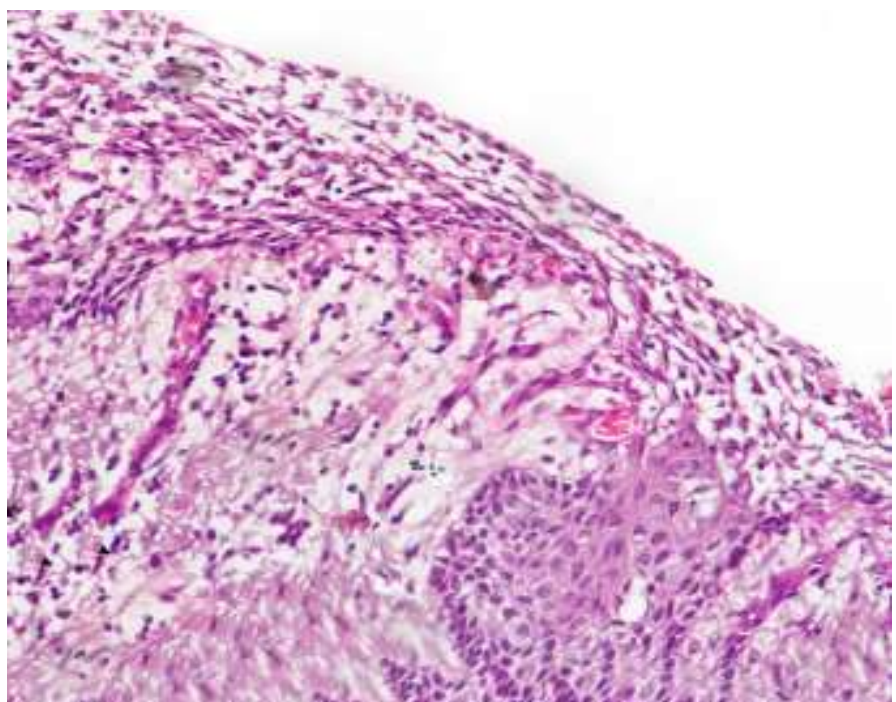

Fig 9 :The capsule consisted of fibrous connective tissue presenting hyperemic vessels, areas of hemorrhage and inflammatory cell infiltrate. 